\title{
Regards, frayeurs et points de suspension dans L'Oiseau Bleu de Maurice Maeterlinck
}

\author{
Sight, fear and suspension points in \\ Maeterlinck's L'Oiseau Bleu
}

\author{
Françoise Paulet Dubois \\ Université d'Almérie \\ pauletfranc@yahoo.fr
}

\begin{abstract}
The very famous play L'Oiseau Bleu, by the Belgian Literature Nobel Prize Maurice Maeterlinck, has been of significant interest for more than a century among theatre and film directors, art creators of all kinds and critics. Its different facets of fairy, symbolic and metaphysical tale make it a charming and surprising play. Examining the vocabulary, I'll study the importance of sight and fear in this work: the words that describe these two notions are superabundant. After trying to find out their meaning for the author, I'll analyse an unusual formal characteristic in the text: the countless suspension points.
\end{abstract}

\section{Keywords}

sight, fear, children, soul, magic.

\begin{abstract}
Resumen
La célebre obra de teatro L'Oiseau Bleu del Nobel belga de literatura Maurice Maeterlinck suscita desde hace más de un siglo un enorme interés por parte de realizadores y directores de cine, creadores de todo tipo y críticos. Sus distintas facetas como cuento de hadas mágico, simbólico y metafísico hacen de este texto una obra entrañable y sorprendente. A partir del léxico, me propongo estudiar la importancia de la vista y del miedo en el texto, ya que los términos para designar estas dos nociones son extremadamente abundantes. Trataré de descifrar los valores que encierran para el autor, y después examinaré una característica formal poco corriente, la multiplicidad de los puntos suspensivos, señal de una preocupación que merece ser analizada.
\end{abstract}

\section{Mots-clés}

vista, miedo, niños, alma, magia. 


\section{Introduction}

Écrite en 1908, étrennée la même année à Moscou par Stanislavski et jouée ensuite inlassablement un peu partout dans le monde, la pièce de théâtre L'Oiseau bleu invite depuis toujours à la rêverie et la réflexion. L'histoire qu'elle raconte a été portée plusieurs fois à l'écran et a inspiré un opéra créé à New York ainsi qu'une série télévisée et des dessins animés japonais. Il s'agit à la fois d'une “'féerie' dont l'essor théâtral coïncidera avec l'attribution du prix Nobel" (Quaghebeur, 2012, I: 5) et d'un conte philosophique. Ce chefd'œuvre fit délirer le public quand Maeterlinck visita les États-Unis, et marqua Faulkner au point que, dans son récit de 1927 intitulé The Wishing Tree, "The details of the myriad birds and talking tree are Maeterlinck's"' (Putzel, 1985: 172), et que l'un des personnages, un petit garçon, se prénomme même Maurice. Enfin, l'intérêt que ce texte suscite de nos jours encore est attesté entre autres par la publication en Suisse, en mars 2018, d'une analyse du théâtre de Maeterlinck.

Au-delà de la poésie aimable et des symboles de ce conte plaisant pour les enfants et admiré par tant d'adultes, je voudrais souligner l'importance du regard, d'une part, et de l'autre celle des frayeurs dans L'Oiseau bleu, à partir d'une approche lexico-sémantique des termes évoquant la vue et la peur. J'examinerai également une curieuse caractéristique du texte: la surabondance de points de suspension.

Au risque de paraître effectuer une étude myope de "la pièce la plus célèbre de Maeterlinck" (Otten, 2012: 175), j'ai choisi pour mon analyse une présentation "rectiligne": elle a du moins le mérite d'accompagner les héros pas à pas dans leur cheminement. Je consacrerai le premier volet à une lecture de l'œuvre, attentive en particulier au vocabulaire du regard et de la peur.

Qu'il me soit permis tout d'abord de considérer L'Oiseau bleu comme une sorte de conte merveilleux théâtralisé, ces deux facettes étant intimement liées. Dans son essai Las raices históricas del cuento, Propp décrit de la façon suivante la trame du récit: "pone en escena a una familia [...] la familia vive feliz y tranquila [...] pequeñísimos e imperceptibles acontecimientos [...] de repente, inesperadamente, desencadenan la catástrofe ${ }^{3 "}$ (1987: 45). On constate ensuite l'absence des parents ou, comme ici, des enfants, frappés d'une interdiction (pour Tyltyl et Mytyl, celle de se lever). Leur désobéissance signalera le début des événements: en effet le petit garçon invite sa sœur à enfreindre la règle, et après la rencontre avec la Fée, ils entreprennent ensemble leurs pérégrinations et leur quête. La peur est un élément important de la narration. Voici ce qu’en pense Propp: “El substrato religioso más

1 J'ai utilisé pour cette étude l'édition de 2012: Espace Nord-Fédération Wallonie Bruxelles. Préface de Marc Quaghebeur; postface de Michel Otten.

2 “Les détails des myriades d'oiseaux et de l'arbre qui parle sont de Maeterlinck" (traduction de l'auteure).

3 "Il met en scène une famille [...] la famille vit heureuse et tranquille [...] des événements insignifiants, imperceptibles [...], tout à coup, de manière inattendue, déclenchent la catastrophe" (traduction de l'auteure). 
antiguo de nuestro motivo está constituido por el temor a las fuerzas invisibles que rodean al hombre" (1987: 59). Même s'il n'y a pas de malheur ou d'accident dans L'Oiseau bleu, ni de motif religieux, les frayeurs de Tyltyl et Mytyl et d'autres personnages, sur lesquelles je m'attarderai, sont bien réelles. Les aventures qu'ils vont courir en des contrées inconnues, ainsi que l'objet de leur recherche, un oiseau parfaitement bleu, cadrent aussi avec les observations du linguiste russe: "La inmensa mayoría de las empresas tiende a mandar al héroe al reino lejano" (1987: 458); "se exige de él que consiga objetos o rarezas que sólo allá se pueden encontrar"' (1987: 459).

Un autre point commun entre le schéma de Propp et la pièce de Maeterlinck est l'existence d'un donneur, un personnage bienfaisant qui fournit au héros le moyen magique de mener à bien son entreprise, et dont l'image est par ailleurs associée aux représentations de la mort. "Su forma clásica es la maga", affirme Propp (1987: 69). La magicienne de notre texte est la fée Bérylune; l'objet enchanté qu'elle offre aux enfants est un chapeau orné d'un Diamant permettant de voir, et elle proclame que "tous ceux qui accompagneront les deux enfants, mourront à la fin du voyage" (36) ${ }^{8}$. De plus, comme l'affirme Borie, "la croyance aux fées [...] figure toutes les puissances de l'imagination" (2018: 68).

Ce conte de fées, cette histoire merveilleuse est chez Maeterlinck théâtralisée, dans le sens où les enfants assistent, et activement, à un spectacle, de connaissance et de reconnaissance. En ce qui concerne le spectateur ou le lecteur, il se trouve face à un théâtre au second degré, à une représentation dans la pièce. Il entre même en jeu, puisqu'à la fin, Tyltyl demande au public de lui rapporter l'oiseau s'il le trouve. Quelle est donc pour Maeterlinck l'importance du théâtre? Partant d'un entretien de l'auteur avec le journaliste Brisson en 1896, Borie affirme que "son attente face au théâtre est [...] 'd'entrevoir un moment la beauté, la grandeur et la gravité de mon humble existence quotidienne”" (2018: 32). On ne peut mieux évoquer l'essence du théâtre, mot dont les racines grecques renvoient à l'idée de contemplation, de visible.

La pièce à peine amorcée, au premier tableau de l'acte premier, Maeterlinck nous indique que "Les deux enfants (...) poussent les volets" de la cabane et "regardent avidement au-dehors" (21); pour ce faire il utilise un verbe formé à partir de "garder", qui signifiait à l'origine "veiller, prendre garde" (Le Petit Robert). Tyltyl, qui contemple l'arbre de Noël, se moque de sa sœur qui regarde le mur. Aux multiples formes du verbe "voir" s'ajoute l'inter-

4 "Le substrat religieux le plus ancien de notre motif est constitué par la crainte des forces invisibles qui entourent l'homme" (traduction de l'auteure).

5 "L'immense majorité des entreprises tendent à envoyer le héros dans un royaume lointain" (traduction de l'auteure).

6 "On exige de lui qu'il obtienne des objets ou des choses bizarres qu'on ne peut trouver que là" (traduction de l'auteure).

7 "Sa forme classique est la magicienne" (traduction de l'auteure).

8 Pour toutes les citations extraites de L'Oiseau bleu, je n'indiquerai entre parenthèses que la page. J'ai d'autre part respecté l'italique pour les didascalies. 
vention du garçonnet, qui enjoint à Mytyl de se taire, de regarder les joujoux et de savourer des yeux les friandises des enfants riches.

Cette plaisante activité est brusquement interrompue par des coups frappés à la porte: les didascalies montrent Tyltyl "effrayé" (étymologiquement, que l'on a fait "sortir de la paix") et Mytyl "épouvantée" (24) (qui ressent "une peur violente et soudaine causée par quelque chose d'extraordinaire, de menaçant", selon Le Petit Robert). Une petite vieille effrayante apparaît qui ressemble à leur voisine Berlingot: c'est la Fée Bérylune. Ce nom, très maeterlinckien, associe la lune et le béryl, mot dérivé du grec $\beta \dot{\eta} \rho \bar{v} \lambda \lambda o \varsigma$, désignant une aigue-marine de couleur bleue ou verte, et qui a donné, entre autres en néerlandais, le substantif bril qui signifie "lunettes". Le nom de la Fée prélude aux actions qui vont suivre, marquées par la vision et par la découverte. Le sursaut est de courte durée et le dialogue s'engage facilement. Elle leur demande "l'oiseau qui est bleu" et chausse "ses bésicles pour examiner" (25) la tourterelle des petits. Le verbe employé a une origine métaphorique, puisqu'il vient d'un mot latin qui veut dire "peser", le terme examen signifiant "aiguille de la balance", selon le Dictionnaire latin-français de Goelzer. L'inspection n'étant pas concluante, les enfants devront aller chercher l'Oiseau Bleu pour la petite fille de la fée, malade de n'être pas heureuse. En route ils pourront "revoir" leurs grands-parents et leurs frères et sœurs défunts. Ici, par l'intermédiaire du préfixe "re-" servant comme dit Le Petit Robert à indiquer "le retour à un état antérieur", la vue est au service de tendresses familiales que les enfants sont pressés de renouer. À partir de là, diverses formes de "voir" émaillent encore les pages 27, 28 et 29: ce pullulement (20 occurrences sur trois pages à peine) anime une discussion entre la Fée, par moments irritée, et Tyltyl, sur la capacité visuelle du petit garçon, et se produit tandis que Bérylune procure aux enfants un chapeau agrémenté d'un Diamant ${ }^{9}$ permettant de voir l'âme des choses. Ce talisman les fera entrer sans cérémonie dans un monde magique. La possibilité d'un retour en arrière visuel se confirme; de même la vision du futur est permise, et la discrétion est assurée puisque personne ne pourra voir le chapeau tant que Tyltyl en sera coiffé.

Dès que l'enfant tourne la pierre précieuse, un prodige s'accomplit: la Fée, les différents personnages, les animaux, la cabane et les objets les plus divers, et même des abstractions comme les Heures, tout prend un aspect merveilleux. Ces métamorphoses provoquent chez l'enfant un "effarement légitime", c'est-à-dire qu'il est troublé, qu'il "ressent un effroi mêlé de stupeur", selon Le Petit Robert; Bérylune, embellie aux yeux dessillés de Tyltyl, le calmera. Mais, alors qu'objets et éléments exhibent leur âme dans un joyeux tourbillonnement, des coups sont de nouveau frappés à la porte, ce qui ravive les craintes du gamin. Comprenant qu'il s'agit de son père, il tourne la pierre magique, mais trop brusquement, et les êtres magnifiquement animés ne parviennent pas tous à recouvrer leur forme originale et regagner leur place. C'est la panique: le Pain "éperdu”, ("profondément troublé par une émotion violente", comme dit Le Petit Robert) est "incapable de rentrer dans la huche et pousse "des rugisse-

9 Comment ne pas évoquer ici "la fée au chapeau de clarté” de Mallarmé dans Apparition, poème de 1887 ? 
ments d'épouvante" (35) et le Feu émet "des sifflements d'angoisse" (36) (un "malaise [...] caractérisé par une crainte diffuse pouvant aller de l'inquiétude à la panique", lit-on dans le même dictionnaire), parce qu'il ne retrouve plus la cheminée. Les coups à la porte redoublent tandis que la Fée et toute la troupe sortent par la fenêtre. Le Père finit par entrer. Les dernières répliques de ce premier acte montrent clairement la connaissance charnelle, extérieure, que les parents Tyl ont de leurs enfants: le Père les voit dormir, et leur présence est confirmée par la Mère, qui les entend respirer. La perception, visuelle et aussi, comme dans ce cas, auditive, des premiers, ne se trouve donc pas sur le même plan que celle des seconds, toute spirituelle. Comme le dit le critique théâtral argentin Dubatti, ils n'ont pas accès au monde métaphysique.

Alors qu'au début de l'acte I, le décor était des plus modestes, l'acte II fait place à la rutilance. Nous sommes à la veille de Noël: les prodiges sont possibles. Les personnages, humains et autres, se sont déguisés d'une manière colorée en empruntant dans la lumineuse garde-robe de la Fée des costumes de contes pour enfants. Tout ce monde est bien visible, affublé de ces toilettes voyantes et, dans les dialogues comme dans les didascalies, l'auteur multiplie les expressions de couleur: l'or à lui seul apparaît trois fois en deux pages (39-40). Une discussion assez vive s'engage entre les partisans et les détracteurs de l'Homme, dieu pour les uns, tyran pour les autres car, comme le déclare la Chatte, "s'il trouve l'Oiseau Bleu, il saura tout, il verra tout, et nous serons complètement à sa merci” (43).

La Fée finit par convaincre l'assemblée de prendre la route sous la direction de la Lumière. Pour elle, "Il est possible que l'Oiseau Bleu se cache dans le Passé" (45): voilà pourquoi la première visite aura lieu chez les grands-parents défunts. La vue va de nouveau jouer un rôle primordial et, à cette occasion, on assiste à un regain des formes de "voir", au futur simple et au futur proche, puisque ces répliques se situent avant la rencontre. Elles transportent une idée maeterlinckienne très émouvante, une leçon simple et belle, celle du souvenir qui nie la mort: "tu vas voir que les morts dont on se souvient vivent aussi heureux que s'ils n'étaient point morts [...]” (47), dit la Fée. Elle pourrait s'exclamer, à l'instar du narrateur d'Aurélia, publié en 1855: “Quel bonheur de songer que tout ce que nous avons aimé existera toujours autour de nous!" (Nerval, 1961: 229). Pendant tout le troisième tableau, au Pays du Souvenir, les enfants en feront l'aimable expérience.

Après avoir traversé une brume épaisse, ils retrouvent Grand-Papa et Grand-Maman Tyl, remplis du joyeux pressentiment que Tyltyl et Mytyl vont arriver. Au cours de la cérémonie des retrouvailles, les grands-parents prétendent qu'une simple pensée des vivants les réveille et qu'alors ils les revoient. Aux yeux des enfants, "tout est plus beau" (54) dans le Pays du Souvenir, jusqu'au merle qui s'avère être bleu. Tyltyl met l'oiseau dans sa cage, convaincu que “c'est lui, l'Oiseau bleu” (55). Le caractère symbolique de la rencontre est résumé dans la formule "c'est une telle fête quand votre pensée nous visite" (60). Malgré leur statisme, dont témoignent les nombreuses négations ${ }^{10}$, c'est d'êtres bien vivants qu'est

10 Aux pages 56 et 57, par exemple, on en trouve à plusieurs reprises avec "pas, plus, rien, jamais, plus rien." 
peuplé le passé: la double vue des enfants a transformé la pensée en chaleureuse présence. Mais, comme les fantômes qui apparaissent et disparaissent au gré du magicien, les êtres chers s'évanouissent et l'oiseau perd sa couleur. La crainte renaît.

L'acte III a d'abord pour cadre le Palais de la Nuit. D'après la Chatte, c'est là que demeure l'Oiseau Bleu, chez les oiseaux des songes ${ }^{11}$. Mais pour ces êtres ailés, la vue de la lumière équivaut à la mort. Notre oiseau symbole du bonheur vivrait donc au sein des rêves. Le récit Aurélia ne s'ouvre-t-il pas sur l'idée que "Le Rêve est une seconde vie" (Nerval, 1961: 219)? On se souvient aussi du conseil du grillon de Florian: "Pour vivre heureux, vivons caché". La Chatte, dans sa lutte contre l'Homme, propose de faire aux enfants "une telle peur qu'ils n'oseront pas insister ni ouvrir la grande porte du fond [...] Les secrets des autres cavernes suffiront [...] à les terrifier" (65), c'est-à-dire les remplir d'une peur paralysante. Elle prétend recourir à la peur comme stratégie pour empêcher les petits de découvrir les oiseaux. C'est la Nuit qui, malgré un premier refus, donnera à Tyltyl les clefs donnant accès à tous les maux et les mystères de l'univers, non sans lui réclamer "le signe"; l'enfant lui répondra: "Voyez le Diamant" (67).

La salle des Fantômes remplit les visiteurs d'un effroi que traduisent didascalies et répliques. Si la caverne des Maladies donne lieu à une pointe d'humour ("Elles ont l'air bien malades vos Maladies» (71), dit Tyltyl à la Nuit), la salle des Guerres est horrible ${ }^{12}$. Pourtant, le garçonnet s'obstine à tout voir, même les Ténèbres et les Terreurs, voilées, timides mais terrifiantes. Ensuite il entrouvre l'épouvantable porte des Mystères; le Silence est un géant terrible, mais Tyltyl poursuit vaillamment une quête où regards et frissons se succèdent. Si les Étoiles, Parfums et Rosées de la Nuit qui se pressent à la porte suivante ravissent les enfants puis disparaissent, le dernier espace, celui où Tyltyl une fois de plus soupçonne que se cache l'Oiseau Bleu, est le lieu des plus grandes "horreurs" (77), mot associé à l'idée d'atrocité, de chose qui inspire effroi et répulsion. La Nuit le conjure de ne point y pénétrer et lui annonce qu'elle-même s'éloignera s'il s'entête à vouloir en ouvrir la porte. Nous sommes ici dans l'un de ces "sombres sites archaïques de l'ancienne vision du monde", comme dit Quaghebeur (2012, I: 7). Mytyl "pousse des cris de terreur inarticulés" ("peur extrême qui bouleverse, paralyse»); on voit le Pain "claquant des dents" ("avoir peur", d'après $\mathrm{Le} \mathrm{Pe}$ tit Robert) puis "fuyant éperdument" (77) (d'une manière folle, extrême). Tous courent se mettre à l'abri, sauf le fidèle Chien, pourtant "haletant et hoquetant d'épouvante contenue", qui accompagne son cher Tyltyl. Quand le garçonnet introduit la clé dans la serrure s'élève "un cri d'épouvante" (78). Dans ce passage, c'est aux effets physiques secondaires de la peur qu'il est fait allusion, et Maeterlinck ne lésine pas sur l'expressivité.

Tout à coup, un spectacle merveilleux se présente à la vue: un jardin de rêve où volent des myriades d'oiseaux bleus. La scène est féerique. Pas d'ornithophobie, pas de scène de

11 Charlier parle de la pièce comme d'une œuvre "à la fantaisie ailée et souple" (1938: 76).

12 Prémonition de l'auteur? Nous sommes en 1908; la Première Guerre mondiale, la Grande Guerre, éclatera quelques années plus tard. 
panique hitchcockienne ${ }^{13}$ : Tyltyl et Mytyl attrapent des oiseaux qui, hélas, meurent les uns après les autres. Tyltyl se cache pour pleurer; la Lumière le console: “tu n'as pas pris celui qui peut vivre en plein jour [...] Nous le retrouverons" (80).

En dépit des adversités, l'enfant ne perd pas courage et poursuit sa mission, cette fois dans la Forêt. Sur l'ordre de la Chatte, Tyltyl tourne le Diamant et l'âme des arbres surgit. Un dialogue plein de poésie et très humain, parfois teinté d'humour, s'établit entre le Peuplier, le Tilleul, le Saule et d'autres arbres des forêts de Belgique. Proust ne qualifie-t-il pas Maeterlinck de "Virgile des Flandres" (Maeterlinck, 1963: 4ème de couverture)? C'est le moment aussi de rappeler le rôle d'intermédiaire entre la terre et le ciel que Propp attribue à l'arbre dans les contes russes: "el cuento ruso refleja la representación ampliamente difundida según la cual los dos mundos (y a veces también los tres, el subterráneo, el terrestre y el celeste) están vinculados por el árbol"14 (1987: 309). Dans la pièce, les âmes des arbres, magiquement dénudées, vont faire le pont entre les petits Hommes et un monde mystérieux. Le Chêne vénérable, sur l'épaule duquel est perché l'Oiseau Bleu, leur tient toutefois un discours "écologique" menaçant. Il sait que les enfants cherchent à percer un secret qui mettrait en danger tous les arbres, déjà victimes des tout-puissants humains. C'est pourquoi le Chêne propose leur mise à mort. D'abord unis contre les enfants, les arbres font néanmoins défection l'un après l'autre et le Chêne, indigné, comprend qu'il devra agir seul. Tyltyl sort son couteau, et les compagnons végétaux du vieil arbre s'efforcent de le retenir. Citons ici Bachelard pour qui «l'arbre, être statique par excellence, reçoit de notre imagination une vie dynamique merveilleuse» (1943, 266). Il ajoute: “Comme l'imagination dynamique l'adore, cet être toujours droit, cet être qui ne se couche jamais!» (1943: 266). Toutefois, la rêverie bachelardienne est aux antipodes de la découverte de Tyltyl, à qui un talisman a fait constater la violence des arbres. L'essayiste réfère par ailleurs à Lawrence, dont je citerai les phrases originales à propos de l'arbre: “A vast individual life, and an overshadowing will. The will of a tree. Something that frightens you ${ }^{15}$ ". C'est plutôt cette volonté d'anéantissement que l'on observe chez le Chêne de Maeterlinck: il appelle à la rescousse les Animaux qui, à l'exception du Chien toujours loyal, s'offrent à le seconder dans son désir de tuer les petits hommes. Mais Tyltyl, avec son arme, déploie sa bravoure et protège Mytyl, qui de peur "pousse des cris aigus" (95). "Pris de panique" (substantif qui renvoie à Pan, le dieu "chez qui, à l'origine, dominent nettement l'animalité et la sauvagerie", selon le Dictionnaire de la mythologie grecque et romaine, et "qui passait pour troubler, effrayer les esprits", comme dit Le Petit Robert) à la vue du couteau, le Cheval prend la fuite, mais toutes les autres bêtes vont se liguer pour attaquer les petits. Ceux-ci hurlent de détresse (96), pour Le Petit Robert encore

13 Le film The Birds d'Alfred Hitchcock à partir d'une nouvelle de Daphne du Maurier, date de 1963.

14 "Le conte russe reflète la représentation largement répandue selon laquelle les deux mondes (et parfois aussi les trois, souterrain, terrestre et céleste) sont unis par l'intermédiaire de l'arbre" (traduction de l'auteure).

15 "Une vaste vie individuelle, et une volonté qui éclipse tout. La volonté d'un arbre. C'est quelque chose de terrifiant" (traduction de l'auteure). 
ce "sentiment [...] d'impuissance que l'on éprouve dans une situation difficile et angoissante (besoin, danger, souffrance) ". Malgré le secours du bon Tylô, la lutte est inégale et cruelle, et tout serait perdu sans la Lumière, qui recommande aux enfants de tourner le Diamant de façon à ne plus voir ces marques d'hostilité. Elle affirme que l'Homme est complètement seul. La Chatte profite de la confusion générale pour attirer la pitié des enfants en déclarant qu'elle a été blessée par le Bœuf; Mytyl lui répond: “Je ne t’ai pas aperçue” (100), en usant d'un verbe qui signifie, pour Le Petit Robert, "distinguer, après un effort d'attention, et plus ou moins nettement."

La descente au cimetière se produit au quatrième acte. C'est dans l'une des tombes que, selon Bérylune, se trouve l'Oiseau Bleu. La Lumière suggère aux enfants de tourner le Diamant pour voir les morts quand il sera minuit. Pain, Sucre et Lait pâlissent, chancellent ou se taisent; la Chien et la Chatte tremblent ou claquent des dents: à différents degrés, la peur est là; mais la Lumière est catégorique: seuls iront chez les morts les enfants, qu'elle tranquillise d'un 'il n'y a rien à craindre" (104), verbe signifiant à l'origine "trembler."

Le Septième Tableau est à nouveau parsemé de formes de "voir". Sous l'action du diamant magique, à partir de minuit, les morts seront visibles. Cette étape dans la déambulation des enfants est inévitable puisque dans les contes merveilleux, et l'on revient à Propp, "todo el rito de la iniciación era considerado como estancia en el país de la muerte ${ }^{16 " ~(1987: ~}$ 524). L'effroi gagne les deux enfants; pourtant "Il n'y a pas de morts" (109). Pour Otten, et quoi que dise Andrieu du "goût de la mort" (1962: 25) contracté par Maeterlinck au collège Sainte-Barbe, "Le message [...] est clair: il n'y a pas de mort; les terreurs que peuvent nous occasionner la pensée de la mort ou la menace des morts reposent sur un complet malentendu". La mort ne serait “qu'un simple passage, un transfert à l'intérieur de l'âme du monde" (2012: 184). Pour ce critique, Maeterlinck viserait à "dédramatiser les terreurs de la mort, [...] la ramener à une apparence» (2012: 185). Ce que les enfants souhaitaient et en même temps redoutaient n'est pas arrivé: ils ne voient pas de morts; ni la mort ni les morts n'existent. De plus, leur persévérance est récompensée: il leur est donné de contempler un spectacle d'innocence, beauté et harmonie, celui, explique la Lumière, de "toutes les joies, tous les Bonheurs des Hommes" (109). Quand elle prévient le groupe que certains Bonheurs sont pires que les Malheurs, le Pain, terrorisé, est qualifié par le Chien de "poltron" (110), mot issu "de l'italien poltrone, 'poulain', par extension 'peureux", d'après le Dictionnaire étymologique Larousse. Malgré tout, à la demande de la Lumière, il accompagnera les enfants, le Chien et le Sucre dans leur visite. Elle-même entrera voilée afin que les Bonheurs n'aient "rien à redouter" (111), c'est-à-dire, comme dit Le Petit Robert, "craindre comme très menaçant." Ce curieux amalgame de frayeur et de bonheur nous rappelle la fragilité et la courte durée des moments heureux.

Le Jardin des Bonheurs est un espace dominé par la pourpre, l'or, l'argent, les pier-

16 "Tout le rite de l'initiation était considéré comme un séjour au pays de la mort" (traduction de l'auteure). 
reries et les brocarts. Là s'agitent des personnages ventripotents et terre-à-terre, dont la Lumière interdit aux enfants d'accepter les présents: ils ne doivent pas oublier leur tâche. Pour faire honte à tous ces gros Bonheurs ne songeant qu'à l'orgie, la Lumière ordonne au garçon de tourner le Diamant, ce qui met à jour leur laideur: cette nouvelle clarté fait cesser la mascarade. Déshabillés par le regard magique, ils sombrent dans la terreur et s'enfuient chez les Malheurs. Surpris de se trouver désormais dans un merveilleux jardin, et grâce à la Lumière, les petits "découvrent" (120) de nombreux petits Bonheurs, "découvrir" ayant pour sens premier dans Le Petit Robert "dégarnir de ce qui couvre". Les Bonheurs des enfants ne détiennent pas l'Oiseau Bleu, mais d'autres Bonheurs s'avancent: ces joyeux personnages reconnaissent les enfants qui, eux, ne les identifient pas parce qu'ils ne les ont jamais vus auparavant: la maison est pleine à craquer de tous ces Bonheurs quotidiens mais, et le chef des Bonheurs leur en fait le reproche, ils ne s'étaient rendu compte de rien. Ces Bonheurs sont naturellement beaux car "c'est tous les jours dimanche, dans toutes les maisons, quand on ouvre les yeux" (123). On songe au secret du renard dans Le Petit Prince de Saint-Exupéry, paru en 1943: “On ne voit bien qu'avec le cœur. L'essentiel est invisible pour les yeux” (1999: 76). C'est ensuite au tour des Grandes Joies de s'approcher, mais Tyltyl et Mytyl ne les reconnaissent pas. La Joie-sans-égale-de-l'amour-maternel demande à ses enfants déconcertés de la regarder. Quand enfin ils reviennent de leur surprise, ils constatent la beauté et l'élégance de leur maman pour la première fois de leur existence. Le dialogue qui suit, entre 1'Amour de Mère ${ }^{17}$ et Tyltyl, jusqu'à l'arrivée de la Lumière, contient un nouveau chapelet d'expressions visuelles axé sur "la vérité" (128).

La rencontre de certaines Joies avec la Lumière est elle aussi particulièrement émouvante: quand enfin elles font sa connaissance, elles cherchent à être reconnues mais avouent les limites de leur vue, qui ne va pas plus loin que leurs "ombres" ou que leurs "songes" (131). Dans leurs effusions à l'adresse de la Lumière, elles multiplient les impératifs. "Voyez", même si ce n'est qu'une formule, est exprimé trois fois dans une seule réplique, où on lit aussi: "Écartez donc ces voiles qui nous cachent encore les dernières vérités et les derniers bonheurs." Toutes ces exhortations impliquent l'idée de voir, ou mieux, l'invitation à voir.

À peine le jeune garçon a-t-il eu le temps de voir les Joies émues aux larmes qu'il est transporté, au cinquième acte, dans le Royaume de l'Avenir. Le visuel se manifeste ici, pour décrire enfants à naître et décors, au moyen d'une déclinaison de nuances de la couleur bleue $^{18}$. La Lumière pronostique de nouveau la découverte de l'Oiseau Bleu, cette fois dans le Royaume des enfants qui ne sont pas encore venus au monde. La conversation qui suit, entre la Lumière, Tyltyl et les Enfants Bleus, regorge de formules évoquant le regard et la vue. $\mathrm{Au}$ jeu innocent de questions et réponses entre les enfants futurs et l'enfant présent s'ajoute

17 On évoquera ici un autre Belge universel, Maurice Carême, qui dédia à sa mère de nombreux poèmes, et même un recueil complet, Mère, en 1935.

18 “Azur", "saphir", "turquoise”, "lapis-lazuli”, "bleu intense”, "azuré” (133); "bleu surnaturel et lumineux”, "azur plus pâle et diaphane" (134); "tournoiement céruléen", "bleuâtres vapeurs" (141). 
l'insistante proposition des petits de montrer au grand leurs machines, idées et inventions. Certains d'entre eux tremblent de quitter leur Royaume. Quant aux deux petits amoureux que le Temps doit séparer car ils ne peuvent pas naître en même temps, ils vivent un drame qui se traduit par une négation poignante: "Je ne le verrai plus" (151). Pour eux, être ensemble, c'est jouir du plaisir de se voir. Alors qu'il disparaît avec les enfants du futur, qui bientôt admirent la beauté de la Terre, le Temps est fâché de surprendre Tyltyl, Mytyl et la Lumière et il les menace, mais le Diamant magique les protégera en les dissimulant aux yeux de l'ennemi.

Au cours du l'acte sixième, les enfants et leurs compagnons reviennent à la maison d'où ils étaient partis la veille de Noël: Tyltyl a d'abord du mal à reconnaître sa demeure mais la Lumière va l'éclairer pour ensuite, tristement, quitter les enfants car sa mission est terminée. Ils ont fait leur possible, mais l'existence de l'Oiseau Bleu est douteuse. L'Eau, le Feu et le Pain font également leurs adieux avant de retourner à leur vie de silence. "Vos yeux vont se fermer à la vie invisible des choses mais je serai toujours là" (158), explique le Pain. Tylô et Tylette ne pourront plus leur parler non plus. Tous promettent aux enfants qu'ils resteront à leur portée et ils les supplient de chercher à les reconnaître et à les comprendre ${ }^{19}$. La pierre précieuse, qui a dévoilé à Tyltyl et Mytyl le véritable visage des êtres, a en outre dilaté le temps: une nuit est devenue un an. Tous les participants savaient d'avance qu'à l'issue du voyage leur vie prendrait fin, métaphoriquement, car leurs âmes cesseraient d'être visibles; c'est pourquoi la durée du périple s'est prolongée. Pour suivre la pensée de Dubatti, je dirai que l'on passe d'une nuit réelle à une année symbolique.

Le décor final est un calque en plus gai de celui du premier tableau. L'émotion et l'incrédulité se partagent l'esprit des enfants, comme le montre ces mots de la mère Tyl à son fils: "Qu'as-tu donc à me regarder comme un émerveillé?" (165). Les petits évoquent des aventures auxquelles les parents ne comprennent rien. La voisine Berlingot, qui pour Tyltyl et Mytyl n'est autre que la Fée Bérylune, prétend que ces divagations ressemblent aux "songeries" (169) qui affectent sa petite fille: incapable de marcher, elle est persuadée que la tourterelle de Tyltyl lui ferait du bien. L'univers de ce dernier a basculé: tout est plus beau, plus grand, plus neuf, et l'oiseau est en train de devenir bleu. Le garçonnet le donne de bon cœur à la fillette, qui guérira miraculeusement. Tyltyl est "émerveillé" (172) de la beauté de la petite et du prodige qui s'est opéré. Les enfants sont émus, heureux, mais tout d'un coup l'oiseau s'enfuit. Ceci nous fait méditer sur le côté éphémère du bonheur, susceptible de s'envoler sitôt qu'est prononcé son nom, ou sur l'amertume de sa perte, comme dans la phrase de Prévert: "J'ai reconnu le bonheur au bruit qu'il a fait en partant".

Les enfants sont revenus au point de départ. Tout leur est familier, et l'on pourrait croire que la boucle est bouclée; pourtant, tout a changé: même si l'Oiseau Bleu ici aussi leur échappe, l'expérience de la nuit qui a duré une année a fait mûrir les enfants. Grâce à

19 Cette vision maeterlinckienne libérée qui débouche sur la vérité ne diffère pas de la vue considérée comme "mode de connaissance", "opération intellectuelle sollicitant un certain nombre de facultés capables de donner accès à un savoir" (Jay-Robert, 2014: paragraphe 13) apparaissant chez Aristophane (Vème siècle av. J.-C.). 
la Lumière, qui a éveillé les yeux de leur cœur, et à la Fée, qui leur a octroyé de voir l'âme des choses, ils ont enfin compris ce qu'Otten appelle l'“inépuisable richesse du quotidien» (2012: 187). Désormais ils regardent autour d'eux autrement et acquièrent une sorte d'omniscience. "'Il faut savoir regarder': telle serait la leçon de L'Oiseau bleu », affirme encore Otten. De plus, si Tyltyl n'a pas l'oiseau en son pouvoir, il est sûr que quelqu'un dans le public le lui rendra: il en va de son bonheur futur.

Son parcours initiatique a été semé d'embûches, mais à aucun moment l'enfant ne s'est rendu. Ceci confirme l'opinion de Bettelheim sur le "message"des contes de fées: "si $[\ldots]$ on affronte fermement les épreuves inattendues et souvent injustes, on vient à bout de tous les obstacles et on finit par remporter la victoire" (1976: 19-20). Même si l'on admet, avec Otten, que "le mystérieux Oiseau bleu [...] se présente [...] comme un leurre» puisque chaque fois que l'enfant le retrouve, "la prise est suivie d'une déception immédiate» (2012: 177), la quête n'est pas infructueuse car on perçoit la confiance et l'optimisme de Tyltyl: la fin de l'histoire, ouverte, est teintée d'espérance. Voilà l'une des raisons pour lesquelles, sans doute, on peut dire que L'Oiseau Bleu est une pièce née "avec le signe de la grâce" (Touchard, cité par Otten, 2012: 175).

À serrer le texte de près, à l'écrémer pour ainsi dire, on constate une surcharge du lexique de la vue. Si Tyltyl et Mytyl ont un atout indiscutable, le chapeau garni d'un diamant qui montre ou cache selon la manière dont on le tourne, l'auteur dispose du langage: il répète à satiété des termes du champ sémantique de la vue, en particulier "voir" dans tous ses états (y compris les présentatifs "voici” et "voilà" ou l'adjectif "visible", qui eux aussi le renferment), "regarder", “apercevoir" ou "découvrir". Ce foisonnement atteste l'importance capitale de l'idée de vue dans L'Oiseau Bleu et correspond aux fonctions qu'elle y remplit. Plutôt que de prétendre dévoiler les intentions de l'auteur, on peut tenter de cerner les valeurs, à cheval sur le réel et le fantastique, transmises par ces expressions réitérées. Un bref récapitulatif démontrera la polyvalence pour Maeterlinck de ce sens fondamental.

Dès le début de l'histoire, les enfants sont doués d'une prédisposition visuelle favorable puisqu'en les contemplant de loin, ils jouent à manger les gâteaux des riches, et déjà ils les dégustent. Le regard leur octroie donc une connaissance et une joie, car ils parviennent à se donner l'illusion qu'ils assistent eux aussi à la fête.

Une fois qu'ils ont reçu l'aide de la Fée, de leurs yeux rallumés par le diamant, ils peuvent appréhender l'intérieur des choses, et ce passage se fait tout naturellement: "la clef essentielle du voir des enfants est ici l'imagination", comme l'affirme Borie (2018: 68). Maintenant doués d'un pouvoir de seconde vue, je dirais de voyance, ils entrent en contact avec les âmes véritables. Voir, et de surcroît voir à l'insu des autres, est bien un privilège fantastique: c'est ainsi par exemple qu'il est donné à Tyltyl de rencontrer l'âme du Chien: désormais délivrée, celle-ci accompagnera jusqu'à la fin son jeune maître.

De même, pourvus de la capacité exceptionnelle de voir vivants des êtres morts, ils 
retrouvent avec émotion leurs grands-parents et leurs frères et sœurs décédés: comme ils avaient pensé avec tendresse à ces chers disparus, l'action de voir a permis une résurrection momentanée.

Réjouissance, la vue est aussi un péril: dans la Forêt, le précieux don apporte aux enfants la révélation d'une sorte de méchanceté universelle, la brutalité foncière des Arbres et des Animaux. Voir désenchante et détrompe. La Lumière leur ouvre les yeux sur le mal existant dans le monde, et sur la solitude qu'implique la condition humaine.

Grâce à la double vue, on apprend, on connaît ou l'on reconnaît, mais on démasque aussi: on découvre "la vérité des choses" (119). La Vérité, maître mot: sa contemplation, pour saint Thomas d'Aquin, serait la plus haute réussite de l'homme; on y parvient par la raison mais aussi par le cœur, nous dit Pascal, ou par les sens, comme le prétend Lucrèce. Dans le cas de nos jeunes héros, un tour de Diamant métaphorique arrache le bandeau et des yeux et du cœur.

Cet apprentissage de la vue par le biais des sentiments est primordial pour la reconnaissance de l'amour maternel. Comme le dit Borie, "Ce voir nouveau va [...] transformer le regard de l'enfant sur l'amour maternel et le bonheur qu'il procure" (2018: 69). Il a en outre un effet thérapeutique, puisque la vue de l'oiseau guérit la fillette de Berlingot.

L'apothéose a lieu au moment où Tyltyl et Mytyl, sortant de leur étrange sommeil, revoient l'humble cabane familiale, leurs parents et les personnes et les objets dont ils ont capté l'âme: ils exultent. N'ont-ils pas des yeux neufs? Ce qu'Otten appelle le "regard transfigurateur" (2012: 188) est devenu la clé pour ces retrouvailles. La vue nouvelle les a transformés: on parle avec cet auteur de "transmutation" (2012: 188). Les épisodes vécus par les petits, parfois féeriques, mais aussi poignants ou terrifiants, n'appartiennent pas à la vie surprotégée d'enfants à qui l'on cacherait les maux et la laideur du monde. Ils ont vu. La vue n'est pas ici un thème littéraire décoratif, mais la composante essentielle et le moteur du conte. Borie ne consacre-t-elle pas plusieurs paragraphes au "voir des enfants" (2018: 68-69) dans L'Oiseau bleu, dans son essai Le théâtre de Maurice Maeterlinck? Charles Péguy disait que la signification du voyage ne nous est pas donnée par la découverte de nouveaux paysages, mais par leur contemplation d'un œil différent.

Au cours de ce périple où la vue prédomine et où la peur est plus d'une fois sur le point de freiner les héros, je me suis attachée, en tant que philologue, aux aspects lexicaux et littéraires de ces notions. Mais on peut ouvrir de nouvelles voies si on les met en rapport avec d'autres disciplines telles que le symbolique, le mysticisme, la poétique, les Beaux-Arts, la sociologie ou la psychanalyse.

Dans L'Oiseau Bleu, le regard incessant est tantôt esthétique, tantôt affectif, spirituel ou cognitif. L'action physique pure et simple de voir s'enrichit de valeurs nouvelles par la médiation d'un objet ayant la particularité d'être à la fois très visible et d'aider à bien voir: un diamant merveilleux. De leurs yeux nus les enfants ne font que l'expérience oculaire; 
avec la pierre magique, ils acquièrent la conscience et apprennent la Vérité des choses et des êtres; c'est grâce à ce "fluide" aussi qu'a lieu la métamorphose de la tourterelle de Tyltyl en un oiseau bleu capable de procurer le bonheur.

Analysant la féerie de Maeterlinck, Katia Vandenborre affirme que, pour un artiste qui croit à "l'unité spirituelle entre le visible et l'invisible", "l'art se doit de déjouer les apparences visibles des choses pour en pénétrer l'essence" (2009: paragraphe 3). Le conte maeterlinckien, dit-elle, est "un produit de l'inconscient, de l'intuition, du fond de l'âme" (2009: paragraphe 4). La morale du bonheur quotidien qui se dégage de la pièce, où en effet la joie de la vérité est donnée à ceux qui savent voir au-delà du réel, boit aux sources de traditions spirituelles et symbolistes. Dans son travail Ruusbroec, en son temps et dans les siècles, Benoît Beyer de Ryke évoque le rôle joué par le mystique flamand du XIVème siècle “dans l'évolution de [Maeterlinck] vers le symbolisme» (2012: paragraphe 2). Notre écrivain, qui traduisit en français une partie de l'œuvre de l'Admirable, y avait trouvé "une forme de symbolisme à l'état originel" (2012: paragraphe 33). Pour Beyer de Ryke, ce mystique d' "inspiration augustinienne" "accorde une grande place à l' amour comme vecteur de la relation entre l'homme et Dieu' (2012: paragraphe 15). Dans L'Oiseau Bleu, même si Dieu n'est pas cité, c'est avec les yeux de la bonté et d'un amour retrouvé que Tyltyl et Mytyl regardent autour d'eux. Ils parviennent ainsi à une sorte de béatitude.

La Poétique du regard de Pierre Ouellet dit de la littérature qu'elle "est [...] connaissances croisées avec des sensations" (2000: 8). Selon lui, elle nous invite à "une expérience perceptive médiate, dont le support est imaginaire, puisque construit à partir d'images mentales résultant de notre double connaissance de la langue et du monde, en jeu dans toute lecture et toute écriture" (2000: 9-10). Si l'on accepte ces idées, on affirmera sans se tromper que la sensation et la perception privilégiées dans l'œuvre littéraire qui nous occupe relèvent du visuel. Dans ce monde-là, la lumière est bien sûr une compagne indispensable. C'est pourquoi on souscrira à la pensée de Bachelard qui, lisant Novalis, la considère comme un feu idéalisé: "Elle attend l'œil. Elle attend l'âme. Elle est donc à la base de l'illumination spirituelle" (1949: 180). La Lumière de Maeterlinck est une précieuse alliée, une éclaireuse prodigue en conseils et en consolation. Elle profère une vérité éternelle: “Ceux qui m'aiment et que j'aime me retrouveront toujours" (105) et nous renseigne sur la psychologie des différents bonheurs. Symbole de prudence, sagesse et mesure, elle se voile quand il le faut, mais sait aussi percer les apparences. Enfin, elle annonce aux Joies qu'elle reviendra "sans craintes et sans ombres" (131): l'assurance ira de pair avec une clarté sans faille.

Un élément de notre conte qui impressionne Tyltyl est le Silence. Or, et nous revenons à Bachelard, "il semble que, pour bien comprendre le silence, notre âme ait besoin de voir quelque chose qui se taise" (1942: 215 20). Le philosophe ajoute: "Maeterlinck a travaillé aux confins de la poésie et du silence, au minimum de la voix, dans la sonorité des eaux

20 À propos de Pelléas et Mélisande. 
dormantes" (1942: 215-216). Dans L'Oiseau Bleu, c'est la vision de cette chose taciturne qui sans doute a manqué à Tyltyl pour qu'il comprenne l'agressivité du silence. Ce qu'il a vu au contraire sans le reconnaître, et cela justifie sa frayeur, c'est une espèce de monstre "sans yeux" (74).

Pour avoir une preuve supplémentaire des qualités profondément visuelles de $L^{\prime} O i$ seau Bleu, il suffit de lire la méticuleuse description du décor et des costumes dès le début et tout au long de l'œuvre. La mise en scène est primordiale. Antonin Artaud écrira que «les possibilités de réalisation du théâtre appartiennent tout entières au domaine de la mise en scène, considérée comme un langage dans l'espace et en mouvement" (1964: 68). Un aspect qu'il convient de relever à ce sujet est la présence des Beaux-Arts. La longue introduction du Neuvième Tableau, Le Jardin des Bonheurs, évoque avec force détails la somptuosité, la brillance et les couleurs de "la Renaissance vénitienne ou flamande (Véronèse ou Rubens)», nommée expressément (112). L'inventaire presque technique des colonnes, draperies, meubles, vaisselle et habits, démontre l'intérêt et les connaissances artistiques de l'auteur. Dans son article sur les rapports de Maeterlinck avec les arts plastiques, le professeur Laoureux montre que "Maeterlinck fut en contact avec de nombreux plasticiens" (2012: paragraphe 3) et que plusieurs de ses écrits “conservent la trace d'une réflexion sur les beaux-arts" (paragraphe 4); il signale aussi "'inspiration picturale dont Maeterlinck a fait preuve pour la conception des décors et des costumes de ses drames" et "l'activité artistique déployée pour les représentations de ceux-ci" (paragraphe 5). Outre qu'elle aide à planter le cadre de l'action, cette verve picturale favorise la représentation symbolique des derniers arrivés, en l'occurrence les gros Bonheurs, images du matérialisme vulgaire. On pourrait parler ici de “symbolisme visuel”, pour reprendre l'expression de Laoureux (paragraphe 16).

Il y a quelques années, Maxime Boidy a rapatrié en France les Visual Studies du monde anglo-américain sous le nom d'“études visuelles", un courant de recherche multidisciplinaire centré sur le problème du visible et de la visibilité. Si nous admettons avec lui que “irréductible à une sensorialité 'naturelle' ou physiologique, le regard est une capacité acquise, inculquée, qui varie selon les époques et les positions sociales" (2017: 65), verrons-nous le singulier regard enfantin qui balaie L'Oiseau Bleu soumis à une analyse sociologique?

La richesse de l'éventail lexical de la peur est elle aussi évidente dans ce petit livre de Maeterlinck. Les frissons d'épouvante le parcourant ne font qu'en rehausser le charme, même si, comme l'affirme Andrieu, "son symbolisme célèbre la fin des terreurs" (1962: 79). Les enfants et leurs compagnons font plusieurs fois l'expérience, physiologique et instinctive, psychologique aussi, de la crainte, de la frayeur, de l'épouvante ou de la panique. L'intensité des peurs qui assaillent les héros est variable, comme aussi leurs causes.

Au commencement de l'histoire, Tyltyl et Mytyl sursautent en entendant des coups frappés à la porte, car ils pensent qu'il s'agit de leurs parents: ceux-ci représentent une autorité à laquelle leurs désirs et leur curiosité les ont fait se soustraire. La crainte ressentie, assez 
commune, est réaliste: dans la peur des parents entrent le respect et un certain sentiment de culpabilité.

L'aveu de frayeur de Mytyl face à l'inexplicable phénomène du changement de couleur d'un oiseau, outre qu'il pimente un peu l'aventure, permet sans doute aux petits lecteurs ou spectateurs de se sentir légitimés quand eux-mêmes éprouvent ce sentiment, dont parfois les grands se moquent. Maeterlinck, qui avait ce qu'Andrieu appelle "le don d'enfance" (1962: 23), manifeste son empathie pour les plus jeunes.

Dans le Palais de la Nuit, la fillette avoue de nouveau son effroi, et le Pain est paniqué au point d'aller se cacher dans le fond de la salle puis de s'enfuir en hurlant: cette peur du noir, de l'inconnu et des Spectres est bien compréhensible, même si de nos jours l'engouement pour Halloween qui parcourt le globe semble l'avoir sublimée! Et si Tyltyl est épouvanté par les Guerres, ceci donne à Maeterlinck l'occasion de montrer par contraste l'héroïsme du garçonnet. Même tremblant et plein de méfiance, il continue à explorer les lieux et s'effraie alors des Ténèbres et autres Mystères et du Silence: ces peurs-là abondent dans les films d'horreur, remplis d'obscurité, de fantômes et de dragons, si prisés du public.

Étoiles et Parfums, de leur côté, redoutent au plus haut point le rayon de soleil qui les ferait mourir. Tyltyl, obéissant aux consignes de la Lumière, surmonte ses appréhensions et, en compagnie de son chien mort de peur, se prépare à franchir une porte interdite. Sa vaillance est couronnée de succès, puisque l'abîme sans nom que défendait la Nuit est un éden où évoluent des milliers d'oiseaux bleus, malheureusement destinés à périr dès qu'ils atteindront le plein jour.

Tout en craignant Tyltyl et son couteau, les âmes des Arbres et des Animaux font les bravaches dans la Forêt. Le Cimetière impressionne les enfants, surtout Mytyl, terrorisée à l'idée de voir des morts, mais l'endroit se métamorphose en jardin comme par enchantement; toute frayeur s'estompe, et le retour dans la chaumière se produit sans heurt.

Présenter des épisodes de terreur répond à un usage habituel dans les contes de fées ou les légendes, et nombreux sont les enfants que la lecture et la tradition ont familiarisés avec l'Ogre, le Grand Méchant Loup ou le père Fouettard. Littérature, cinéma, déguisements et jeux divers fourmillent d'événements et de monstres terribles: ceci rend compte de l'intérêt d'un vaste public pour les sensations qui donnent la chair de poule. Nombre de tout-petits même raffolent de l'expérience de la peur: la preuve en est leur goût pour le jeu qui consiste à les surprendre par un "bouh!" et les faire sursauter, surtout s'ils ont la certitude que des bras bienveillants les attendent pour les rassurer et les consoler. Dans la pièce de Maeterlinck, outre qu'il donne de la vivacité à l'histoire en faisant alterner inquiétude et plaisir, et fournit un contrepoint aux émerveillements, ce recours à des situations effrayantes met en relief le sens du devoir de Tyltyl, ainsi que son instinct de protection envers sa sœur: il contrôle sa peur pour mener à bien sa mission.

Qu'est-ce que la peur? Naturelle et universelle, la peur est une sensation indispen- 
sable à la survie de l'humanité: si nos ancêtres avaient été moins effrayés face à certains dangers, ils auraient pu être exposés aux blessures, aux mutilations, voire à la mort.

Sous l'angle de la psychanalyse, c'est "un système de défense, d'alarme, face à un danger éprouvé par le moi”, comme dit Joël Bernat, reprenant la pensée de Freud. Cet affect est une "élaboration psychique" de l'effroi, lui-même défini comme une «réaction de détresse psychique du moi face à une situation de danger" (2018: 1) imprévue et en cela traumatisante. Cette élaboration se produit par "l'attribution d'un objet défini au danger effrayant", ce qui met l'effroi à distance, et place "entre le moi et le danger [...] la représentation d'un objet ou d'une situation dangereuse." L'angoisse quant à elle est un affect qui prépare au danger, "une prévention de la menace"; l'angoisse est donc "une défense naturelle" (2018: 2).

L'horreur mérite une mention à part. Aline Estèves, qui a consacré sa thèse de doctorat à la poétique de l'horreur dans certains textes latins, distingue "l'horreur au sens objectif" de "l'horreur au sens subjectif": si la première a un contenu variable en fonction des individus et des circonstances, la seconde "se laiss[e] identifier [...] comme une peur extrême et exceptionnelle", et son "imaginaire psycho-physiologique" inclut "épouvante mêlée de haine ou d'admiration, aphasie, paralysie, panique, hérissement de la pilosité et traumatisme émotionnel persistants". (2006: 28).

Ayant violé l'interdiction de leurs parents, les petits héros de la pièce, confrontés à différents dangers, feront la désagréable expérience de toutes ces sensations, à des moments et des degrés divers. Gageons que Maeterlinck n'y soumet ses personnages que pour mieux accentuer leur courage et leur détermination. Ces épreuves traumatisantes atteignent donc un caractère légendaire, puisqu'elles servent de repoussoir aux qualités des enfants: ils ont affronté bien des périls, mais ils ont surmonté ou dépassé leurs terreurs, et ils ont survécu; mais surtout, ils ont acquis de nouvelles dimensions émotionnelles et spirituelles. Le père Tyl, qui occupe d'ailleurs un espace bien maigre dans la pièce, prend pour des balivernes le récit du mystérieux voyage de ses enfants; il veut les guérir en leur donnant des claques mais, grâce à la voisine, ils échappent à la punition. À ce propos, revenons à Bettelheim, pour qui “ce n'est pas un hasard si l'enfant, au moment où il est [...] déçu par ses parents qui cessent de répondre à ses attentes infantiles, devient physiquement et psychiquement capable de satisfaire lui-même certains de ces besoins" (1976: 193). Parfois, c'est un «autre personnage" (dans notre "conte", la Fée, et à travers elle, la "pensée magique" à laquelle Borie fait allusion $^{21}$ ) qui permettra au héros de "corriger la mauvaise opinion qu'ils ont de lui" (1976: 208). Dans L'Oiseau bleu, le nouveau regard, la lucidité nouvelle, fait mûrir Tyltyl et Mytyl et éteint leurs angoissses.

Après le regard et la peur, un trait formel du texte, moins superficiel sans doute qu'il n'en a l'air, a retenu mon attention: il est criblé de points de suspension. Quel est leur utilité dans la pièce?

21 "le Gros diamant [...] fait voir [...] au-delà des apparences [...] mais c'est avant tout l'ouverture des enfants à la pensée magique qui a rendu possible l'accès à un autre voir, du monde et des êtres" (Borie, 2018: 68). 
Pour Julien Rault, qui dans sa thèse consacrée à la poétique du point de suspension ${ }^{22}$ se livre à une étude extrêmement fouillée de la ponctuation en général, "la principale difficulté [...] repose sur l'extrême variété des descriptions et interprétations possibles" (2014: 215). Il affirme aussi que le point fondamental relatif à ce ponctème est "l'investissement du lecteur" (2014: 213). Il ajoute que "le point de suspension s'oppose évidemment à l'idée d'un dire plein, abouti, à l'imaginaire d'une communication transparente. Il exhibe le caractère non-plein du dire, se pose comme contraste au dire plein" (2014: 277). On rejoint ici Colignon, quand il affirme, dans un essai qui se veut un guide, que les points suspensifs, actuellement au nombre de trois, “indiquent que la pensée n'est pas complètement exprimée, que la phrase est laissée en suspens -volontairement de la part du scripteur- ou bien, que s'agissant d'un tiers ou d'un personnage littéraire en particulier, qu'un événement extérieur vient interrompre le propos ou l'action" (2004: 101). Enfin, et pour en arriver à la suite de points dans une pièce de théâtre, je rappellerai les mots de Conruyt (2018): “à l'origine, ces caractères ont une fonction précise et ce, uniquement dans le domaine théâtral”; ils représentent "textuellement une indication scénique"; ils agissent comme des "didascalies graphiques"ou "silencieuses" (Hawcroft, 2006: 315).

Remarquons que, dans cette œuvre de Maeterlinck, il ne se produit pas d'aposiopèse, ou interruption du discours. Aucun personnage ne coupe la parole à l'autre comme c'est le cas par exemple dans le théâtre de Racine. La prolifération de ce signe à travers toutes les pages a plutôt le don d'aérer le texte, de le rythmer et de laisser aux personnages, partant, aux acteurs, le temps de reprendre haleine.

L'itération du point dans L'Oiseau bleu figure en fin de phrase et est quasiment toujours suivie d'une majuscule. Les phrases ainsi "prolongées" sont aussi bien énonciatives (affirmatives ou négatives) qu'interrogatives ou exclamatives. En voici un échantillon: "Ils sont morts aussi [...]"; "Voulez-vous les revoir?[...] "; "Montrez-les![...] "; "Je ne les ai pas dans ma poche $[\ldots]$ "..23 . Les trois points semblent renforcer la pensée ou le sentiment exprimés. À l'occasion simple signe de clôture, le point triplé peut devenir lui-même un marqueur expressif, comme après ces mots de la Fée: "tous ceux qui accompagneront les deux enfants, mourront à la fin du voyage [...]" (36), où l'étirement muet de la phrase suggère une menace voilée, un avertissement. Dans une invitation au regard comme "Regardez donc, ils mangent les rayons de la lune![...]" (79), les points et le silence qu'ils représentent ont la vertu de faire rêver le lecteur ou le spectateur, et d'amplifier le ton poétique. Cela suggère moins l'inachèvement qu'une sorte de chuchotement du locuteur qui ne peut ou ne veut se prononcer et préfère le non-dit.

Charlier évoque, dans le théâtre de Maeterlinck "des silences, des attitudes, de l'attente angoissée et fiévreuse; de là cette expression balbutiante et ces obsédantes répéti-

22 Rault considère qu'il serait plus juste d'employer le "singulier [...] si l'on veut prendre en considération la spécificité du point de suspension, ses valeurs, ses emplois et ses effets propres" (2014: 216).

23 Tous ces exemples sont extraits de la page 27. 
tions" (1938: 75). Borie affirme: "Maeterlinck lui-même, dans son théâtre, ne rêvera-t-il pas d'une œuvre habitée par le silence?" (2018: 14), ou encore: "Le théâtre de Maeterlinck [...] sera sans cesse traversé par cette obsession d'un au-delà des mots et des images ouvrant sur le silence, seul habité par l'essentiel" (2018: 15). Le langage de notre prix Nobel est sobre, et l'émaciation va jusqu'au souffle. Nous sommes bien au-delà des mots. La retenue de l'auteur, au moyen de trois points qui représentent l'ineffable, confirme pour moi l'idée de Borie selon laquelle le théâtre de Maeterlinck est "un théâtre de la dimension cachée" (34). Cette ponctuation inhabituelle, à la présence obsessive d'un bout à l'autre de la pièce, semble enfin attendre du lecteur ou du spectateur qu'il rêve, imagine, construise, à partir de ce qui est tu. Gorceix évoque à ce propos une des exigences de l'esthétique symboliste, qui réclamerait "la contribution nécessaire de l'auditeur, non plus passif dans l'accueil d'un produit fini, mais incité précisément par des paroles inachevées, par le caractère vague et imprécis des dialogues, à compléter ce que l'auteur, lui, n'a voulu que suggérer" (1997: 23). L'“obscur”, pour reprendre le mot de Gorceix, est aussi l'indicible.

Le petit animal fragile et évanescent ayant nom Bonheur, qui s'efface ou change de couleur pour désorienter et mieux s'échapper, ce n'est qu'au prix d'épreuves effroyables, de silencieux mystères et d'un jeu de cache-cache avec la lumière que d'abord on le reconnaîtra et qu'ensuite on tentera de le saisir! Inatteignable comme un amour impossible, ou comme Dieu, ou comme un rêve, cet objet de désir fait pourtant sortir Tyltyl et Mytyl de leur chrysalide d'inconscience enfantine: grâce à un regard neuf sur les êtres et les choses, ils s'ouvriront à l'espoir d'un bonheur tangible.

\section{Références bibliographiques}

AA.VV. 1962. Dictionnaire illustré de la mythologie grecque et romaine. Paris, Seghers.

AA.VV. 1993. Dictionnaire alphabétique et analogique de la langue française 1. Le Nouveau Petit Robert. Paris, Dictionnaires Le Robert.

ANDRIEU, Jean-Marie. 1962. Maurice Maeterlinck. Paris, Éditions universitaires.

ArTAud, Antonin. 1964. Le théâtre et son double. Paris, Gallimard.

Bachelard, Gaston. 1942. L'Eau et les Rêves. Essai sur l'imagination de la matière. Paris, Corti.

Bachelard, Gaston. 1943. L'Air et les Songes. Essai sur l'imagination du mouvement. Paris, Corti.

BaChelard, Gaston. 1949. La psychanalyse du feu. Paris, Gallimard.

Bettelheim, Bruno. 1976. Psychanalyse des contes de fées. Paris, Laffont. Titre original: The Uses of Enchantment. Trad.: Théo Carlier. 
Bernat, Joël. 2018. "Peur: origine et fonction", D'un divan l'autre [consulté le 28 avril 2019]. <www.dundivanlautre.fr/.../joel-bernat-peur-origine-et-fonction>

Beyer DE RyKe, Benoît. 2005 (mis en ligne le 8 juin 2012). "Ruusbroec, en son temps et dans les siècles" in Textyles 28/ 2005,19-29. DOI: 10.4000/textyles.426 [Consulté le 8 mai 2019]. <http://journals.openedition.org/textyles/426>

BoIDy, Maxime. 2017. "Image, vision, visualité" in Les études visuelles. Saint-Denis, Presses universitaires de Vincennes, "Libre cours", 65-82 [consulté le 10 mai 2019]

$<$ https://www.cairn.info/les-etudes-visuelles--9782842927356-page-65.htm>

Borie, Monique. 2018. Le théâtre de Maurice Maeterlinck. Lausanne, Ides et Calendes.

Charlier, Gustave. 1938. Les Lettres Françaises de Belgique. Bruxelles, La Renaissance du Livre.

Colignon, Jean-Pierre. 2004. Un point c'est tout! Paris, Victoires-Éditions.

Conruyt, Claire. 2018. "Séduction, suggestion... Ce qui se cache derrière les points de suspension", Le Figaro.fr langue française. www.lefigaro.fr > Langue Française > Expressions [consulté le 9 mai 2019].<37003-20181212ARTFIG00022-seduction-suggestion-ce-qui-secache-derriere-les-points-de-suspension.php>

Dauzat, Albert; Dubois, Jean; Mitterand Henri. 1964. Nouveau dictionnaire étymologique et historique. Paris, Larousse.

DubatTI, Jorge. 2008. "Maurice Maeterlinck y el drama simbolista (en el Centenario del estreno mundial de El Pájaro Azul)" in Revista del Centro Cultural de Cooperación Floreal Gorini, Palos y Piedras. Edition: 5 / 6, Buenos Aires [consulté le 8 juin 2018].<http://www. centrocultural.coop/.../maurice-maeterlinck-y-el-drama-simbolista-en-el-centena...>

Estèves, Aline. 2006. “Poétique de l'horreur dans l'épopée et l'historiographie latines, de l'époque cicéronienne à l'époque flavienne: imaginaire, esthétique, réception" in L'information littéraire, 2006/2 Vol.58, 27-32 [consulté le 27 avril 2019].<https://www.cairn.info/ revue-1-information-litteraire-2006-2-page-27.htm>

Georgin, Charles. 1957. Dictionnaire grec-français. Paris, Hatier.

Goelzer, Henri. 1928. Dictionnaire latin-français. Paris, Garnier.

GorCEIX, Paul. 1997. "Maeterlinck symboliste: le langage de l'obscur" in Textyles, 1-4,| 1324. DOI : 10.4000/textyles.1643 [consulté le 4 juillet 2018]. <http://textyles.revues.org/1643>

GorCEIX, Paul. 2003. "Maurice Maeterlinck, 'Confession de poète', un texte-manifeste de la création poétique symboliste” in Littératures en contact: mélanges offerts à Vic Nachtergaele. Louvain, Presses Universitaires, 57-64.

HAnse, Joseph. 1983. Nouveau dictionnaire des difficultés du français moderne. Gembloux, Duculot.

Hawcroft, Michael. 2006. "Points de suspension chez Racine: enjeux dramatiques, enjeux éditoriaux" in Revue d'histoire littéraire de la France, Presses Universitaires de France 2006/2 Vol. 106, 307-335 [consulté le 30 avril 2019].<https://www.cairn.info/revue-d-histoire-litteraire-de-la-france-2006-2-page-307.htm> 
JAY-Robert, Ghislaine. 2014 (mis en ligne le 5 juin 2014). "La Vision comme moyen d'accéder à la connaissance et au pouvoir: manipulation du regard chez Aristophane" in Cahiers des études anciennes, 21-44. [consulté le 4 mai 2019].<http://journals.openedition.org/etudesanciennes/788>

LaOureux, Denis. 2000 (mis en ligne le 18 juin 2012). "Maurice Maeterlinck et les arts plastiques: filiations et concomitances" in Textyles, 17-18. DOI: 10.4000/textyles.1419 [consulté le 28 avril 2019]. <http://journals.openedition.org/textyles/1419>

LaWrence, David Herbert. 1922. Fantasia of the Unconscious. New York, Thomas Seltzer. [consulté le11 mai 2019]. <https://beelyrics.net/music/38055-d-h-lawrence/165516-fantasiaof-the-unconscious-chap-4-lyrics.html>

Maeterlinck, Maurice. 1934. Pelléas et Mélisande. Paris, Fasquelle.

Maeerlinck, Maurice. 1963. La Vie des Abeilles. Paris, Fasquelle.

Maeterlinck, Maurice. 1964. El Pájaro azul. Interior. Buenos Aires, Éd. Losada. Trad.: R. Brenes Mesen et Gregorio Martínez Sierra.

Maeterlinck, Maurice. 1980. Euvres: Quinze chansons, Les Aveugles, L'Intruse, Serres chaudes. Bruxelles, Éd. Jacques Antoine.

Maeterlinck, Maurice. 2012. L'Oiseau bleu. Bruxelles, Espace Nord-Fédération Wallonie Bruxelles.

Maeterlinck, Maurice. 2012. La Princesse Maleine. Bruxelles, Espace Nord-Fédération Wallonie Bruxelles.

Nerval, Gérard (de). 1961. Les Filles du Feu. Aurélia. Paris, Gallimard et Librairie Générale Française.

Otten, Michel. 2012. Postface de L'Oiseau bleu de Maurice Maeterlinck. Bruxelles, Espace Nord-Fédération Wallonie Bruxelles.

Ouellet, Pierre. 2000. Poétique du regard: Littérature, perception, identité. Limoges, Presses universitaires; Septentrion [consulté le 11 mai 2019]. <https://www.septentrion.qc. ca/catalogue/poetique-du-regard>

Propp, Vladimir. 1987. Las raíces históricas del cuento. Madrid, Ed. Fundamentos. Titre original: Istoričeskie korni volšebnoj skazki. Trad.: José Martín Arancibia.

Putzel, Max. 1985. Genius of Place: William Faulkner's Triumphant Beginnings. Baton Rouge, Louisiana State University Press.

Quaghebeur, Marc. 1980. Préface des Euvres: Quinze chansons, Les Aveugles, L'Intruse, Serres chaudes de Maurice Maeterlinck. Bruxelles, Éd. Jacques Antoine.

Quaghebeur, Marc. 2012, I. Préface de L'Oiseau bleu de Maurice Maeterlinck. Bruxelles, Espace Nord-Fédération Wallonie Bruxelles.

Quaghebeur, Marc. 2012, II. Préface de La Princesse Maleine de Maurice Maeterlinck. Bruxelles, Espace Nord-Fédération Wallonie Bruxelles. 
Rault, Julien. 2014. Poétique du point de suspension: valeur et interprétations. Poitiers: Université de Poitiers [Consulté le 9 mai 2019]. <theses.univ-poitiers.fr/58120/2014-RaultJulien-These>

SAInt-EXupéry, Antoine de. 1999. Le Petit Prince. Paris, Gallimard.

Touchard, Pierre-Aimé. 1962. "Le Dramaturge” in Maurice Maeterlinck 1862-1962, sous la direction de Joseph Hanse et Robert Vivier. Bruxelles, La Renaissance du Livre.

TLFi: Trésor de la langue Française informatisé. ATILF-CNRS \& Université de Lorraine. [consulté le 9 mai 2019].<http://www.atilf.fr/tlfi $>$

VAndenborre, Katia. 2009. "Analyse comparative de la féerie chez Maeterlinck et Wyspiański. La Noce face au premier théâtre de Maeterlinck" in Revue de littérature générale et comparée [consulté le 9 mai 2019]. $<$ https://journals.openedition.org/trans/348>

Walzer, Pierre-Olivier. 1963. Essai sur Stéphane Mallarmé. Paris, Seghers. 
